\title{
STUDY OF THE POLARIZED STRUCTURE FUNCTIONS OF THE NEUTRON AT LOW $Q^{2}$ WITH POLARIZED ${ }^{3} \mathrm{He}$
}

\author{
Seonho Choi* \\ Temple University \\ 1900 N. 13th Street, \\ Philadelphia, PA 19122, USA \\ E-mail: choi@jlab.org
}

\begin{abstract}
We have measured the electron $-{ }^{3} \mathrm{He}$ longitudinal and transverse spin dependent inclusive cross sections ${ }^{3} \overrightarrow{\mathrm{He}}\left(\vec{e}, e^{\prime}\right)$, mostly in the resonance region (up to $W \simeq$ $2.5 \mathrm{GeV})$, over a wide range of four-momentum transfer $\left(Q^{2}=0.03-1 \mathrm{GeV}^{2}\right)$. The longitudinally polarized electron beam of energy ranging from 0.86 to $5.1 \mathrm{GeV}$ of the Jefferson Lab was scattered at a fixed angle of $15.5^{\circ}$, on a high pressure polarized ${ }^{3} \mathrm{He}$ target in Hall A. The spin dependent structure functions $g_{1}(x), g_{2}(x)$ have been extracted and their moments were evaluated. The GDH integral shows dramatic transition from large $Q^{2}$ domain to low $Q^{2}$ domain and the BurkhardtCottingham sum rule for $g_{2}$ seems to hold within experimental errors. $d_{2}$ is quite large and positive consistent with the SLAC data but at odds with lattice QCD calculation.
\end{abstract}

\section{Introduction}

The study of the spin structure of the nucleon has been of central interest in the last few decades. The measurements of $g_{1}$ and $g_{2}$ and their interpretation are one of the key tests for QCD. In the DIS region, it has been found that only a small fraction (about 20\%) of the nucleon spin is coming from the spin of quarks and that Bjorken sum rule ${ }^{1,2}$ holds to better than $10 \%$ level. On the other extreme, where $Q^{2}=0$, there is Gerasimov-DrellHearn (GDH) sum rule ${ }^{3,4}$ which is being tested actively at various facilities. These two fundamental sum rules of QCD can be connected via generalized GDH sum rule for intermediate range of $Q^{2}$. While both sum rules involve mainly $g_{1}$ structure function, there is also interesting physics involving $g_{2}$ such as the Burkhardt-Cottingham sum rule ${ }^{6}$ or $d_{2}$ matrix element. In this

${ }^{*}$ For JLab E94010 Collaboration 
manuscript, recent results on the neutron spin structure from Jefferson Lab will be presented.

\section{QCD Sum Rules}

At the limit where $Q^{2} \rightarrow \infty$, the spin structure function $g_{1}\left(x, Q^{2}\right)$ satisfies Bjorken sum rule ${ }^{1,2}$,

$$
\int_{0}^{1}\left[g_{1}^{p}\left(x, Q^{2}\right)-g_{1}^{n}\left(x, Q^{2}\right)\right] d x=\frac{1}{6} \frac{g_{A}}{g_{V}}
$$

where $g_{1}^{p}(x)$ and $g_{1}^{n}(x)$ are spin structure functions for the proton and the neutron respectively, while $g_{A} / g_{V}$ is a ratio of vector and axial coupling constant. This sum rule, originally shown using current algebra has been re-derived using the technique of operator product expansion (OPE) within QCD.

On the other limit at $Q^{2}=0$, there is GDH sum rule ${ }^{3,4}$,

$$
\Gamma_{1}\left(Q^{2} \rightarrow 0\right)=-\frac{Q^{2}}{8 M^{2}} \kappa^{2}
$$

where $\kappa$ is the anomalous magnetic moment of the nucleon.

Recently, it has been shown ${ }^{5}$ that these two sum rules at both extremes of $Q^{2}$ can be connected via generalized GDH sum rule,

$$
\Gamma_{1}\left(Q^{2}\right)=\frac{1}{8} Q^{2} S_{1}\left(Q^{2}\right)
$$

where $S_{1}\left(Q^{2}\right)$ is the forward virtual Compton scattering amplitude which, in principle, can be evaluated for every value of $Q^{2}$. The Bjorken sum rule is a natural consequence of this generalized GDH sum rule by taking difference of $S_{1}\left(Q^{2}\right)$ between the proton and the neutron at high $Q^{2}$.

For the structure function $g_{2}\left(x, Q^{2}\right)$, another interesting sum rule has been derived by Burkhardt and Cottingham ${ }^{6}$,

$$
\Gamma_{2}\left(Q^{2}\right)=\int_{0}^{1} g_{2}\left(x, Q^{2}\right) d x=0 .
$$

This sum rule holds for every value of $Q^{2}$ but does not follow from the OPE. Its validity relies on $g_{2}\left(x, Q^{2}\right)$ obeying Regge behavior at low $x$ and there exists several possible scenarios which can lead to the violation of the $\mathrm{BC}$ sum rule $\mathrm{e}^{7,8,9}$. 
Another interesting quantity which can be studied with precision data for $g_{2}(x)$ is the $d_{2}$ matrix element defined as

$$
\begin{aligned}
d_{2}\left(Q^{2}\right) & =\int_{0}^{1} x^{2}\left[2 g_{1}\left(x, Q^{2}\right)+3 g_{2}\left(x, Q^{2}\right)\right] d x \\
& =3 \int_{0}^{1} x^{2} \overline{g_{2}}\left(x, Q^{2}\right) d x,
\end{aligned}
$$

where

$$
\begin{aligned}
\overline{g_{2}}\left(x, Q^{2}\right) & =g_{2}\left(x, Q^{2}\right)-g_{2}^{W W}\left(x, Q^{2}\right) \\
g_{2}^{W W}\left(x, Q^{2}\right) & =-g_{1}\left(x, Q^{2}\right)+\int_{x}^{1} \frac{g_{1}\left(y, Q^{2}\right)}{y} d y .
\end{aligned}
$$

Here $g_{2}^{W W}\left(x, Q^{2}\right)$ derived by Wandzura-Wilczek ${ }^{15}$, is twist- 2 contribution to $g_{2}\left(x, Q^{2}\right)$. As a result, $d_{2}\left(Q^{2}\right)$ matrix element is a measure of higher twist effect in $g_{2}\left(x, Q^{2}\right)$ and contains non-trivial quark gluon interactions beyond the parton model.

At high $Q^{2}, d_{2}$ can be expressed as linear combination of the induced color electric and magnetic polarizabilities $\chi_{E}$ and $\chi_{B}$ when a nucleon is polarized $^{13,14}$. At small $Q^{2}$, since the very notion of "higher twist" becomes blurry, the meaning of $d_{2}$ is not clear. However, at very low $Q^{2}$, recent work has shown that $d_{2}$ can be expressed as a linear combination of transverse $\left(\gamma_{0}\right)$ and longitudinal $\left(\delta_{L T}\right)$ nucleon spin polarizabilities ${ }^{10}$.

\section{Experiment}

We have measured the inclusive cross section for the scattering of polarized electrons from a polarized ${ }^{3} \mathrm{He}$ target in Hall $\mathrm{A}$ of the Jefferson Lab. An electron beam with average polarization of $70 \%$ and current up to $15 \mu \mathrm{A}$ was scattered off a high density (10 atm in a $40 \mathrm{~cm}$ long glass cell) polarized ${ }^{3} \mathrm{He}$ target with average $35 \%$ of polarization. The scattered electrons were detected by the two essentially identical spectrometers sitting at $15.5^{\circ}$ on both sides of the beam. Data were collected at six incident beam energies: $5.508,4.239,3.382,2.581,1.718$ and $0.862 \mathrm{GeV}$. The experiment covers a range of $Q^{2}$ from 0.03 to $1.1 \mathrm{GeV}^{2}$, and from the elastic to the edge of the deep inelastic regime, including the quasi-elastic and the resonance regions. Data were taken for both longitudinal and transverse target polarization orientations so that the two spin structure functions $g_{1}\left(x, Q^{2}\right)$ and $g_{2}\left(x, Q^{2}\right)$ can be extracted independently. More details on the experiment can be found elsewhere $e^{11,12}$. 


\section{Results}

The two structure functions $g_{1}\left(x, Q^{2}\right)$ and $g_{2}\left(x, Q^{2}\right)$ have been extracted from measured differential cross sections for each combination of electron and target spin directions. In order to perform integration of structure functions at constant $Q^{2}$, the measured structure functions were interpolated (or extrapolated for a few points slightly out of the actual kinematic coverage) for six equally spaced values of $Q^{2}$ between 0.1 and $0.9 \mathrm{GeV}^{2}$.

After the integration at constant $Q^{2}$, two corrections have been applied: one for the nuclear effects coming from the other two protons in the polarized ${ }^{3} \mathrm{He}$ target and the other for the contribution to the integral from the unmeasured kinematic region.
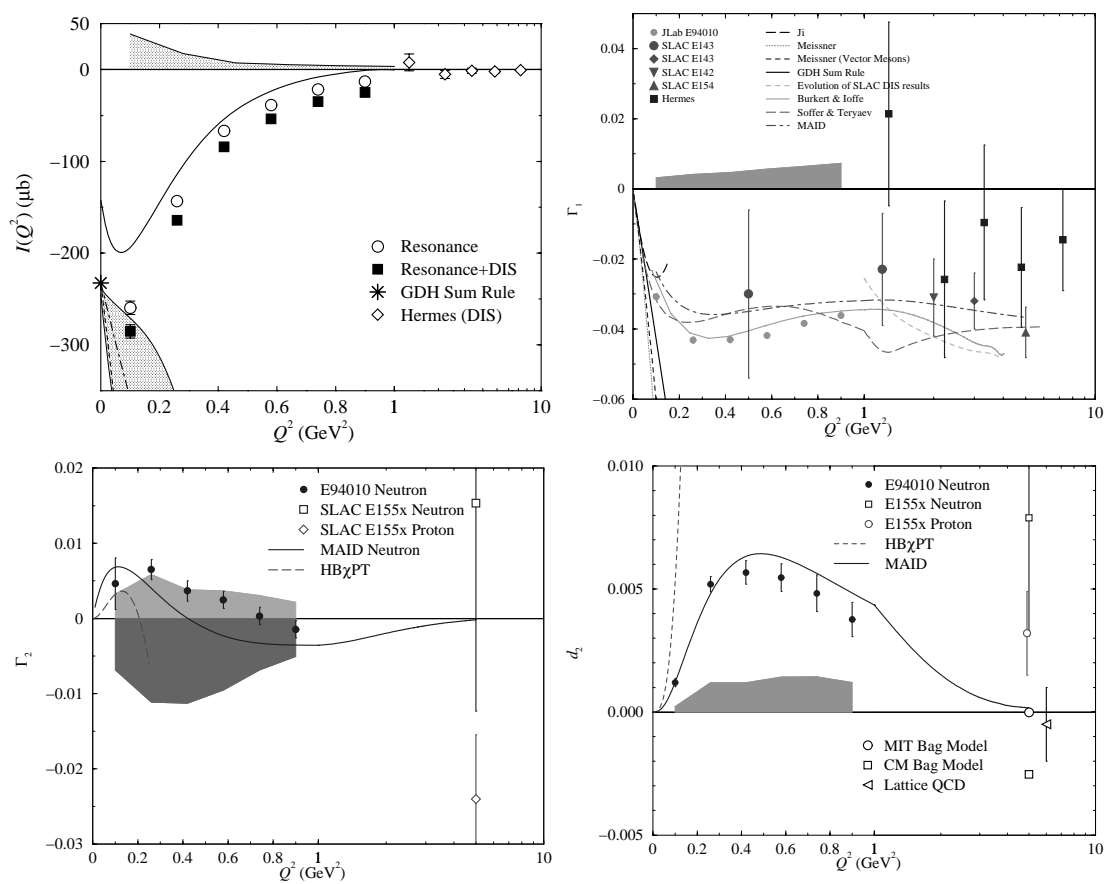

Figure 1. Integral of spin structure functions. The error bars for the present data are only statistical. Systematic errors are shown with shaded band along zero $y$-axis. TopLeft: generalized GDH integral, Top-Right: integral of $g_{1}\left(x, Q^{2}\right)$, Bottom-Left: integral of $g_{2}\left(x, Q^{2}\right)$, Bottom-Right: $d_{2}$ matrix element.

Figure 1 shows the various integrals of spin structure functions from this experiment: generalized GDH integral (top-left), $\Gamma_{1}\left(Q^{2}\right)$ (top-right), 
$\Gamma_{2}\left(Q^{2}\right)$ (bottom-left) and $d_{2}\left(Q^{2}\right)$ matrix element (bottom-right).

The generalized GDH integral shows dramatic change from high to small $Q^{2}$ region approaching real photon GDH sum rule shown by an asterisk at $Q^{2}=0$. The general trend of the GDH integral is well predicted by MAID model but within our measured kinematic region, MAID underestimates the experimental results. Around $Q^{2}=0$, comparison with real photon GDH sum rule suggests a turn over of the trend, but our measured $Q^{2}$ range is not small enough to see this interesting feature. Maybe future experiments ${ }^{16}$ will provide more interesting results in this region. Nevertheless, the overlap of our lowest point with the gray band (the prediction of $\chi \mathrm{PT}$ including certain resonance effects) is quite encouraging and we look forward to further refined calculations at small $Q^{2}$ region.

As shown in the figure, we have provided the first precision data for $\Gamma_{1}\left(Q^{2}\right)$ for the neutron in the $Q^{2}$ range between 0.1 and $0.9 \mathrm{GeV}^{2}$. In this intermediate $Q^{2}$ range, three model calculations are shown. Our measured data agree quite well with that of Burkert and Ioffe. Again MAID model underestimates $\Gamma_{1}$ and Soffer and Teryaev's calculation shows interesting oscillatory feature which was not observed in the our data. Our data $\Gamma_{1}$ on the neutron, when combined with those on the proton from JLab Hall-B will give an evolution of Bjorken sum at small $Q^{2}$ region and will provide another test for QCD.

The integral of $g_{2}\left(x, Q^{2}\right)$ for the neutron shows very interesting result as regard to the Burkhardt-Cottingham sum rule. Our data suggest that the BC sum rule holds within errors over the measured $Q^{2}$ region although the integration has been limited in $x$ range. At high $Q^{2}$ another measurement by SLAC showed a possible violation of the BC sum rule for the proton and inconclusive result for the neutron due to the large error. One more interesting feature is that MAID prediction agrees quite well with measured data in this case and it does not predict a large violation of the $\mathrm{BC}$ sum rule. In this respect, precision data for $g_{2}\left(x, Q^{2}\right)$ for the proton at small $Q^{2}$ range would be very useful for checking the $\mathrm{BC}$ sum rule.

The last frame shows our result of $d_{2}\left(Q^{2}\right)$ with SLAC data and a few available model calculations. As in the case of the integral of $g_{2}\left(x, Q^{2}\right)$, the MAID model agrees pretty well with the measurement. The $\chi \mathrm{PT}$ calculation is divergent at very small $Q^{2}$ and does not give any useful comparison with the data. Our data shows large and positive $d_{2}$ in the range of $Q^{2}$ between 0.1 and $0.9 \mathrm{GeV}^{2}$, but if our data follows the same trend as MAID model, it will decrease to zero at high $Q^{2}$ where SLAC data are available. However, the SLAC data show large and positive $d_{2}$ at odds with the pro- 
jection of our data nor with various theoretical predictions. Here again, precision data of $g_{2}\left(x, Q^{2}\right)$ for $Q^{2}$ between 1 and $5 \mathrm{GeV}^{2}$ will be crucial in discerning apparent contradiction.

\section{Summary and Future}

We have measured spin dependent cross sections and asymmetries for ${ }^{3} \mathrm{He}\left(e, e^{\prime}\right)$ and extracted spin dependent structure functions $g_{1}\left(x, Q^{2}\right)$ and $g_{2}\left(x, Q^{2}\right)$ at $Q^{2}$ range from 0.1 to $0.9 \mathrm{GeV}^{2}$. From these, various QCD integrals have been evaluated for the neutron for the first time for intermediate $Q^{2}$ range. For the GDH integral and $\Gamma_{1}$, general trend of the measurement is well predicted by models and future measurement ${ }^{16}$ at even lower $Q^{2}$ will provide direct comparison with $\chi \mathrm{PT}$ calculation near $Q^{2}=0$. The $\mathrm{BC}$ sum rule for the neutron seems to hold within errors and our result for $d_{2}$ suggests positive sign at high $Q^{2}$ contrary to various theoretical predictions. In this respect, further precision measurements of $g_{2}\left(x, Q^{2}\right)$ for both the proton and the neutron from low $Q^{2}$ up to $5 \mathrm{GeV}^{2}$ are crucial.

\section{References}

1. J. D. Bjorken, Phys. Rev. 148, 1467 (1966).

2. J. D. Bjorken, Phys. Rev. D 1, 1376 (1970).

3. S. B. Gerasimov, Sov. J. Nucl. Phys. 2, 430 (1966).

4. S. D. Drell and A. C. Hearn, Phys. Rev. Lett. 16, 908 (1966).

5. X. Ji and J. Osborne, J. Phys. G 27, 127 (2001).

6. Hugh Burkhardt and W. N. Cottingham, Annals of Physics 56, 453 (1970).

7. I. P. Ivanov et al., Phys. Rep. 320175 (1999).

8. M. Anselmino, A. Efremov and E. Leader, Phys. Rep. 261, 1 (1995); Erratum-ibid, 281399 (1997).

9. R. L. Jaffe and Xiangdong Ji, Phys. Rev. D 43, 724 (1991).

10. Chung Wen Kao, Thomas Spitzenberg and Marc Vanderhaeghen, hep$\mathrm{ph} / 0209241$.

11. M. Amarian et al., Phys. Rev. Lett. 89, 242301 (2002).

12. http://www.jlab.org/e94010.

13. B. W. Filippone and X. Ji, Adv. in Nucl. Phys. 26, 1 (2001).

14. X. Ji, in Proceeding of the Workshop on Deep Inelastic scattering and QCD, Editors: J. F. Laporte and Y. Sirois, Paris, France, 24-28 April, 1995 (ISBN 2-7302-0341-4).

15. S. Wandzura and F. Wilczek, Phys. Lett. B72, 195 (1977).

16. J.-P. Chen, A. Deur and F. Garibaldi, JLab proposal E97110, The GDH Sum Rule and the Spin Structure of ${ }^{3}$ He and the Neutron using Nearly Real Photons. 